\title{
Establishment of the Japanese training system for percutaneous endoscopic lumbar discectomy: from the stand point of neurosurgery
}

\author{
Junichi Mizuno \\ Center for Minimally Invasive Spinal Surgery, Shin-Yurigaoka General Hospital, Kanagawa 210-0024, Japan.
}

Correspondence to: Dr. Junichi Mizuno, Center for Minimally Invasive Spinal Surgery, Shin-Yurigaoka General Hospital, Kanagawa 210-0024, Japan. E-mail: mizuno@shibire.com

\begin{abstract}
How to cite this article: Mizuno J. Establishment of the Japanese training system for percutaneous endoscopic lumbar discectomy: from the stand point of neurosurgery. Mini-invasive Surg 2017;1:103-5.
\end{abstract}

Article history: Received: 5 Apr 2017 Accepted: 18 Apr $2017 \quad$ Published: 30 Sep 2017

Historically, lumbar laminectomy and fusion is the traditional surgical intervention for lumbar degenerative diseases such as herniated discs or canal stenosis with or without spondylolisthesis. Although this procedure was once the most commonly performed technique, serious complications were frequent. The most common complications included instability and severe back pain due to destruction of the biomechanical stability of the lumbar spine. To compensate for these complications, an operating microscope was introduced to the field of spinal surgery in the 1950s. Currently, microdiscectomy, the so-called "micro-Love method" based on the classic technique that Dr. Love reported ${ }^{[1]}$ has been established as the gold standard procedure for herniated lumbar discs. Due to the less invasive approach and the fine surgical manipulations, conventional complications dramatically decreased and surgical outcome have significantly improved.

In the beginning of this century, microendoscopic discectomy (MED) was developed by using a less than $20 \mathrm{~mm}$ tubular retractor. ${ }^{[2]}$ Serial dilators also are used to avoid detachment of the paraspinal muscles from the spinous process. This procedure was considered to be a less invasive, but it still requires the removal of the ligamentum flavum as well as to drill the lamina and the medial portion of the facet joint. Thus, this procedure gained popularity among some spinal surgeons, however the outcomes were not superior to the micro-Love technique. Invasion of the spinal canal by either micro-Love or MED can destabilize the spinal segment and create scarring in and around the spinal nerves. Destruction of soft tissues and bony structures is inevitable when approaching the compressed spinal nerves in micro-Love and MED.

Percutaneous endoscopic lumbar discectomy (PELD) and percutaneous endoscopic lumbar laminectomy (PELL) can avoid a significant portion of approachrelated complications. ${ }^{[3,4]}$ Third generation systems, such as the Yeung Endoscopic Spine System, ${ }^{[5]}$ include a cannula set with slotted openings that allow instruments to exit the cannula for surgical work while a protruding tongue protects and retracts adjacent structures. The beveled cannula allows for visualization of the disc and epidural space simultaneously, thus License (https://creativecommons.org/licenses/by/4.0/), which permits unrestricted use, distribution and reproduction in any medium, as long as the original author is credited and the new creations are licensed under the identical terms. 
facilitating the removal of subligamentous, extruded, and sequestered disc fragments. Foraminoplasty can enlarge the foramen to decompress the spinal canal and lateral recess stenosis.

PELD allows for targeted fragmentectomy of the herniated disc by performing the "inside-out" technique. Unlike the knee or hip joints, the spinal canal has no fluid cavity. Continuous irrigation of the endoscope is necessary. Basic techniques of PELD includes transforaminal approach and interlaminar approach. The route to the spinal canal or spinal nerves is different in these two approaches, and the spinal surgeons must select the best approach in each case. Once the cannula is placed inside the spinal canal, the "inside-out" technique is performed to remove the herniated disc.

In 2009, the first hands-on training course of spinal endoscopic procedure was organized at the 16th Annual Meeting of Japanese Society for Neuroendoscopy (JSNE) in Toyama (http://www.med.u-toyama.ac.jp/nsurgery/jsne2009/). Spinal endoscopic training was officially accepted as one of the neuro-endoscopic training courses by JSNE the following year. In 2014, the first hands-on training course was organized at the 34th Annual Meeting of Japanese Congress of Neurological Surgeons (https://www.jcns-online.jp/en/) in Osaka. In 2016, Neuro-Spinal Society of Japan (NSSJ) approved to start the official training system of PELD and PELL. Japanese Society Orthopedic Surgery had started the official training system prior to our training system, and there are 131 board-certified MED, surgeons and 23 board-certified PELD surgeons on November, 2016. NSSJ initially approved 5 board-certified PELD surgeons, Dr. Junichi Mizuno, Dr. Yasuhiko Nishimura, Dr. Yukoh Ohara, Dr. Yoshihiro Kitahama and Dr. Hisaaki Uchikado to initiate the training system. All of these 5 surgeons have experienced various techniques of PELD and PELL through huge volumes of knowledge and surgical techniques. Interested individuals who want to apply the certification system must be an official member of NSSJ and an official member of Japanese Society of Neurological Surgery. Ten clinical experience of either PELD or PELL as a major operator in the past are required. The lectures and hands-on training courses (both anatomical model and cadaver) are also mandatory to attend. This certification system restricts laser disc decompression or percutaneous endoscopic cervical discectomy. Additional, the member can only apply to this certification system after the number of attendance has reached the specified number of times. The applicants must send the operative summary of previous experiences of PELD and operative video record which is not edited together with their curriculum vitae, recommendation letter by any of previously certified members to the PELD office in Shin-Yurigaoka General Hospital (office.peld@lesnm.ac). An investigation committee which consists of previously certified 5 members has a role of the judgment of the application form and the operative video record. The review process by the committee is closed to the general public but applications are strictly assessed. If applicant does not pass the examination, points for improvement will be conveyed to the applicant.

The learning curve for endoscopic spine surgery is very steep, however it can be grasped by every endoscopic surgeon with proper training. As with novel surgical procedures, the complication rate may be relatively higher during the learning curve. Proper inclusion and exclusion criteria for PELD is the first step for a successful operation. Correlation of the preoperative image and patients' subjective pain must be well understood by the surgeon who performs PELD. For the safer endoscopic operation, the importance of the intraoperative neurophysiological monitoring, anesthesia or surgical tools and techniques should be discussed for the future advances of endoscopic spine procedures.

Finally, several academic meetings related to the minimally invasive spinal operations including PELD or MED are listed below:

Less-Invasive and Endoscopic Spinal Neurosurgery (LESNM)

Japanese Society of Minimally Invasive Spinal Surgery (JASMISS)

Pacific and Asian Society of Minimally Intervention Spinal Surgery (PASMISS)

International Society of Minimally Intervention in Spinal Surgery (ISMISS)

World Congress of Minimally Invasive Spine Surgery and Techniques (WCMISST)

\section{DECLARATIONS}

\section{Authors' contributions}

J. Mizuno contributed solely to this paper.

\section{Financial support and sponsorship}

None.

\section{Conflicts of interest}

There are no conflicts of interest.

\section{Patient consent}

Not applicable. 


\section{Ethics approval \\ Not applicable.}

\section{REFERENCES}

1. Love JG. Protruded intervertebral disc (Fibrocartilage): (Section of Orthopædics and Section of Neurology). Proc $R$ Soc Med 1939:32:1697-721.

2. Perez-Cruet MJ, Foley KT, Isaacs RE, Rice-Wyllie L, Wellington
R, Smith MM, Fessler RG. Microendoscopic lumbar discectomy: technical note. Neurosurgery 2002;51:S129-36.

3. Yeung AT. The evolution of percutaneous endoscopy and discectomy: state of the art. Mt Sinai J Med 2000;67:327-32.

4. Kambin P, O'Brien E, Zhou L, Schaffler JL. Arthroscopic microdiscectomy and selective fragmentectomy. Clin Orthop Relat Res 1998;(347):150-67.

5. Yeung AT. Minimally invasive disc surgery with the Yeung Endoscopic Spine System (YESS). Surg Technol Int 1999;8:267-77. 